\title{
COMPARAÇÃO DOS RESULTADOS DO LIMIAR \\ DE DETECTABILIDADE DE VOZ POR MEIO \\ DE MATERIAL GRAVADO E A VIVA VOZ
}

\section{Comparing the results of lower voice detection thresholds by means of recorded material and live voices}

\author{
Angela Ribas ${ }^{(1)}$, Karlin Fabianne Klagenberg ${ }^{(2)}$, Marine da Rosa Diniz ${ }^{(3)}$, \\ Bianca Simone Zeigelboim ${ }^{(4)}$, Jackeline Martins-Bassetto ${ }^{(5)}$
}

\section{RESUMO}

Objetivo: comparar os resultados do teste denominado limiar de detectabilidade de voz, utilizando-se uma lista de palavras trissilábicas previamente gravadas e à viva voz em indivíduos com audiograma de configuração plana. Métodos: participaram da pesquisa 60 indivíduos (120 orelhas) com exame audiométrico dentro dos padrões da normalidade e audiometria tonal de configuração plana, sem intercorrências auditivas, em faixa etária de 20 a 30 anos. Aplicou-se o teste à viva voz e com material gravado, em cabine tratada acusticamente, por meio do audiômetro AC40 e com o auxílio de um cd player acoplado. Resultados: o limiar de detectabilidade de voz foi encontrado $10 \mathrm{~dB}$ melhor que a média tritonal em 35 orelhas $(58,5 \%$ da amostra) quando realizado a viva-voz e em sete orelhas $(11,5 \%)$ quando realizado com material gravado; e foi encontrado $5 \mathrm{~dB}$ pior que a média tritonal em uma orelha $(1,5 \%)$ quando realizado a viva-voz e em 23 orelhas (38\%) com material gravado. Conclusão: a) ao utilizar-se material gravado para o estabelecimento do LDV em curvas de configuração plana, deve-se considerar como padrão de normalidade níveis de intensidade iguais à média tritonal ou pior em $5 \mathrm{~dB}$ e b) evidenciou-se diferença entre os resultados obtidos com material gravado e a viva voz, o que demonstra a importância da utilização de testes gravados para determinação dos limiares auditivos na logoaudiometria.

DESCRITORES: Audiometria da Fala; Audição; Percepção Auditiva

(1) Fonoaudióloga; Docente do Curso de Graduação em Fonoaudiologia da Universidade Tuiuti do Paraná; Doutora em Meio Ambiente e Desenvolvimento Humano pela Universidade Federal do Paraná.

(2) Fonoaudióloga; Doutoranda no programa de pós-graduação da Universidade Tuiuti do Paraná; Mestre em Distúrbios da Comunicação pela Universidade Tuiuti do Paraná.

(3) Fonoaudióloga; Docente no Centro Universitário de João Pessoa; Mestre em Distúrbios da Comunicação pela Universidade Tuiuti do Paraná.

(4) Fonoaudióloga; Coordenadora do Programa de Mestrado e Doutorado em Distúrbios da Comunicação da Universidade Tuiuti do Paraná; Doutora em Ciências dos Distúrbios da Comunicação Humana pela Universidade Federal de São Paulo.

(5) Fonoaudióloga da empresa de aparelhos Audiotec; Mestre em Distúrbios da Comunicação pela Universidade Tuiuti do Paraná.

\section{INTRODUÇÃO}

Os testes de fala são de grande importância no diagnóstico audiológico e possuem o objetivo de mensurar a habilidade de um indivíduo em perceber e reconhecer os sons da fala ${ }^{1-3}$, englobando tanto medidas de sensibilidade como de acuidade auditiva. Eles vêm sendo muito estudados atualmente, buscando-se desenvolver materiais padronizados, com o intuito de se obter resultados mais confiáveis, que eliminem ao máximo a influência do examinador ${ }^{4}$. A obtenção de dados mais fidedignos nos testes de fala aumenta cada vez mais a credibilidade da avaliação audiológica ${ }^{5}$. No presente estudo, abordou-se a influência da forma de apresentação do sinal acústico, seja à viva voz ou 
por meio de estímulos gravados, sobre os resultados no Limiar de Detectabilidade de Voz (LDV), que mede a menor intensidade com a qual o indivíduo consegue detectar a presença da voz humana, sem necessariamente compreendê-la ${ }^{1}$.

O LDV é realizado, normalmente, quando o indivíduo não consegue responder aos outros testes de percepção de fala. Por exemplo, indivíduos com perdas auditivas severas e profundas ou indivíduos que não apresentam linguagem oral de etiologias diversas. Pode ser utilizado também na confirmação dos limiares tonais e na seleção do aparelho de amplificação sonora individual. No Brasil, o examinador, depois de regular a intensidade do audiômetro, emite uma série de sílabas ou logatomos, utilizando um microfone que envia o sinal ao examinando. Este, por sua vez, deve indicar toda vez que sente o som, até que se determine o nível mínimo de sensação do mesmo ${ }^{6,7}$. Como estímulo o examinador pode utilizar também outros sons onomatopaicos, palavras, frases e textos.

O limiar encontrado deve coincidir com a média dos limiares tonais das freqüências de 500, 1000 e $2000 \mathrm{~Hz}$, no caso de audiogramas de configuração plana, ou com o melhor limiar tonal encontrado, no caso de audiogramas de configuração ascendente ou descendente. Uma variação de $5 \mathrm{db}$ para mais ou para menos em relação à média tritonal pode ser observada, já que na logoaudiometria o estímulo utilizado possui um amplo espectro de freqüência, quando comparado ao tom puro utilizado na audiometria tonal ${ }^{6,7}$.

Os resultados dos testes logoaudiométricos podem variar em função do tipo de material utilizado para avaliação, ou ainda, em função do sucesso do ouvinte em perceber os traços supraliminares da fala. No último caso, ao se comunicar, uma pessoa lança mão, além das pistas segmentares da fala (fonemas conjugados em palavras, palavras conjugadas em frases), as pistas supra-segmentares, isto é, busca auxílio em variações de intensidade e timbre, na articulação e coarticulação, expressões faciais, tipo de material de fala, tema, assunto, fatores temporais e qualidade vocal ${ }^{8}$. Quando se aplica o teste da logoaudiometria, estes fatores mencionados devem ser isolados daqueles segmentares, que devem ser de maior importância durante a testagem, desta forma, para se garantir a padronização do material, listas gravadas com itens de teste devem ser priorizadas. A apresentação do estímulo por meio da gravação aumenta a estabilidade da condição, porém tende a diminuir a flexibilidade da técnica ${ }^{9-11}$.

Existe literatura justificando e priorizando a utilização da logoaudiometria com material gravado e padronizado ${ }^{12}$, em se tratando de apresentação à viva voz, o efeito de diferentes falantes pode afetar a confiabilidade dos testes ${ }^{13,14}$ e a apresentação das mesmas palavras por um único falante variará em diferentes condições ${ }^{15}$. A entonação, o sotaque e qualquer alteração vocal podem interferir nos resultados logoaudiométricos ${ }^{16}$. Desta forma, a qualidade vocal de uma determinada pessoa pode variar, e tal modificação pode ser observada em função da quantidade e qualidade dos harmônicos produzidos nas cavidades de ressonância de seu trato vocal ou do padrão vibratório das pregas vocais por maior ou menor tensão muscular na região glótica.

Na prática clínica não é raro encontrar resultados do LDV variando de -10dBNS a +10dBNS, em relação à média tritonal, em virtude de diferenças relativas à entonação e pronúncia do examinador. Também não é raro, num mesmo paciente, avaliado por dois examinadores diferentes, haver diferença de limiares, tal fato influenciado pela voz do examinador. Assim, o objetivo do presente estudo foi comparar os resultados do LDV, utilizando-se uma lista de palavras trissilábicas previamente gravadas e à viva voz em indivíduos com audiograma de configuração plana.

\section{MÉTODOS}

O estudo foi realizado no Laboratório de Audiologia da Clínica de Fonoaudiologia da Universidade Tuiuti do Paraná (UTP), no ano de 2006. Todos os participantes da pesquisa assinaram o termo de consentimento livre e esclarecido.

Foram avaliados 60 indivíduos voluntários, oriundos da Universidade Tuiuti do Paraná, 37 do sexo feminino e 23 do masculino, num total de 120 orelhas. Os sujeitos enquadraram-se nos seguintes critérios: idade entre 20 e 30 anos, sem história de intercorrências audiológicas e portadores de audiograma de configuração plana bilateralmente. Eles foram divididos, aleatoriamente, em dois grupos, $\mathrm{A}$ e $\mathrm{B}$, cada qual composto por 30 indivíduos. Foram excluídos da pesquisa aqueles que apresentaram perda auditiva.

A avaliação audiológica foi realizada após anamnese e otoscopia, em cabine acústica, utilizando-se o audiômetro AC 40 da marca Interacoustics com fones TDH 39 com limiares expressos em dBNA. Os equipamentos foram devidamente calibrados conforme resoluções 295/03 e 296/03 do Conselho Federal de Fonoaudiologia.

Após realização da audiometria tonal, e constatando-se que o indivíduo submetido ao teste apresentava audiograma dentro dos padrões da normalidade exigidos na configuração plana, aplicou-se posteriormente a logoaudiometria. 


\begin{tabular}{|c|c|c|c|c|}
\hline Ainda & Atenção & Batata & Espaço & Justiça \\
\hline Banana & Cansado & Direção & Família & Melado \\
\hline Cabelo & Cigarro & Escola & General & Natural \\
\hline Caneta & Difícil & Expressão & Janeiro & Perigo \\
\hline Cidade & Encontro & Garagem & Medida & Presente \\
\hline Criança & Exemplo & Igreja & Molhado & Programa \\
\hline Embora & Futuro & Marido & Pequeno & Seguinte \\
\hline Estudo & Idéia & Militar & Possível & Sorvete \\
\hline Formação & Maneira & Parede & Professor & Vontade \\
\hline Idade & Mercado & Posição & Sapato & \\
\hline Laranja & Panela & Problema & Soldado & \\
\hline Menino & Polícia & Revista & Trabalho & \\
\hline Palavra & Primeiro & Sistema & Cabeça & \\
\hline Pipoca & Respeito & Tomate & Camisa & \\
\hline Primeira & Senhora & Bastante & Cenoura & \\
\hline Projeto & Telhado & Boneca & Criação & \\
\hline Segunda & Bolacha & Caminho & Efeito & \\
\hline Tapete & Caderno & Cavalo & Espécie & \\
\hline Bigode & Casaco & Conversa & Farofa & \\
\hline Cadeira & Conjunto & Direito & Governo & \\
\hline
\end{tabular}

Fonte: Harris et all, $2001^{17}$.

\section{Figura 1 - Lista de palavras trissílabas utilizadas na aplicação do LDV}

Para a determinação do LDV utilizou-se uma lista de palavras trissilábicas pois as mesmas fornecem uma condição de escuta realista da comunicação oral diária, como observa-se na Figura $1{ }^{17}$. A mesma lista de palavras foi aplicada aos dois grupos sendo que no grupo $A$ aplicou-se o teste à viva voz e no grupo B aplicou-se o teste com material gravado.

A gravação da lista de palavras em compact disk foi realizada de acordo com a norma ISO 8253-3 ${ }^{18}$. A lista gravada foi apresentada por meio de um CD player acoplado ao audiômetro utilizado no teste.

A intensidade utilizada para a apresentação do estímulo foi de $40 \mathrm{dBNS}$, ou seja, $40 \mathrm{~dB}$ somados à média tritonal obtida a partir dos limiares audiométricos nas freqüências de $500 \mathrm{~Hz}, 1000 \mathrm{~Hz}$ e $2000 \mathrm{~Hz}$. $\mathrm{O}$ examinador utilizou-se da técnica descendente tanto com o material gravado quanto a viva voz até o momento em que o indivíduo obtinha $50 \%$ de acertos.

Os resultados obtidos no teste foram categorizados da seguinte maneira:

- Melhor + $10 \mathrm{~dB}$ : quando o resultado do LDV foi melhor que a média tritonal em mais de $10 \mathrm{~dB}$, por exemplo, a média tritonal do indivíduo estava calculada em $20 \mathrm{~dB}$ e o LDV foi registrado em $5 \mathrm{~dB}$;
- Melhor $10 \mathrm{~dB}$ : quando o resultado do LDV foi melhor que a média tritonal em exatos $10 \mathrm{~dB}$, por exemplo, a média tritonal do indivíduo estava calculada em $20 \mathrm{~dB}$ e o LDV foi registrado em 10dB;

- Melhor $5 \mathrm{~dB}$ : quando o resultado do LDV foi melhor que a média tritonal em exatos $5 \mathrm{~dB}$, por exemplo, a média tritonal do indivíduo estava calculada em $20 \mathrm{~dB}$ e o LDV foi registrado em $15 \mathrm{~dB}$;

- Igual a média: quando o resultado do LDV foi igual à média tritonal;

- Pior $5 \mathrm{~dB}$ : quando o resultado do LDV foi pior que a média tritonal em $5 \mathrm{~dB}$, por exemplo, a média tritonal foi de $20 \mathrm{~dB}$ e o LDV foi registrado em 25dB;

- Pior $10 \mathrm{~dB}$ : quando o resultado do LDV foi pior que a média tritonal em $10 \mathrm{~dB}$, por exemplo, a média tritonal foi de $20 \mathrm{~dB}$ e o LDV foi registrado em 30dB;

O presente estudo foi aprovado pelo Comitê de Ética Institucional sob número UTP 065/05.

Para análise dos dados, comparando-se os resultados obtidos entre os grupos $\mathrm{A}$ e $\mathrm{B}$, aplicou-se o teste estatístico de comparação de proporções. Considerou-se o nível de significância de $p<0,05$. 


\section{RESULTADOS}

Os dados referentes à categorização dos resultados do LDV estão apresentados na Tabela 1.

O grupo A apresentou maior concentração de dados, resposta de 35 orelhas (58,5\%), na categoria melhor $+10 \mathrm{~dB}$, ou seja, quando o resultado do LDV foi melhor que a média tritonal em mais de $10 \mathrm{~dB}$.

Já no grupo B observou-se maior concentração de dados, resposta de 39 orelhas $(65 \%)$, nas categorias igual à média e pior que a média em $5 \mathrm{~dB}$.

Nos dois grupos observou-se similaridade de resposta para as categorias melhor $10 \mathrm{~dB}$, melhor $5 \mathrm{~dB}$ e pior $10 \mathrm{~dB}$.

Os dados comparativos dos resultados obtidos estão apresentados na Figura 2. O teste de comparação entre a proporção aplicado nos Grupos A e B para as categorias reunidas demonstrou que houve diferença significativa.

\section{DISCUSSÃO}

Antes de se iniciar a discussão dos resultados é imprescindível pontuar que quando se trata de logoaudiometria, exame do qual faz parte o teste do LDV, o estímulo apresentado, à viva voz ou em forma gravada, é um som complexo que impressiona as estruturas auditivas periféricas e centrais de diferentes maneiras. As respostas dadas a estes tipos de estímulos, apesar de condicionadas, são dependentes, muitas vezes, das experiências que o indivíduo tem com a linguagem, e desta forma, fica difícil normatizar acerca de limiares que, em alguns casos, não se configuram exatamente como objetivos.

O LDV deve ser encontrado, em curvas audiométricas planas, perto da média tritonal, variando em $5 \mathrm{~dB}$ para mais ou para menos da mesma ${ }^{7,8}$. Neste estudo pode-se observar que o grupo B, composto por indivíduos submetidos ao teste com material gravado, apresentou um desempenho compatível com o estimado pela literatura, pois

Tabela 1 - Número de orelhas em relação às categorias de resposta no LDV

\begin{tabular}{lcccc}
\hline Categorias & \multicolumn{2}{c}{ Grupo A } & \multicolumn{3}{c}{ Grupo B } \\
\hline Melhor $+10 \mathrm{~dB}$ & 35 & $(58,5 \%)$ & 7 & $(11,5 \%)$ \\
Melhor $10 \mathrm{~dB}$ & 10 & $(17 \%)$ & 9 & $(15 \%)$ \\
Melhor $5 \mathrm{~dB}$ & 7 & $(11,5 \%)$ & 5 & $(8,5 \%)$ \\
Igual a média & 6 & $(10 \%)$ & 16 & $(27 \%)$ \\
Pior $5 \mathrm{~dB}$ & 1 & $(1,5 \%)$ & 23 & $(38 \%)$ \\
Pior $10 \mathrm{~dB}$ & 1 & $(1,53 \%)$ & 0 & -- \\
Total & 60 & $(100 \%)$ & 60 & $(100 \%)$ \\
\hline
\end{tabular}

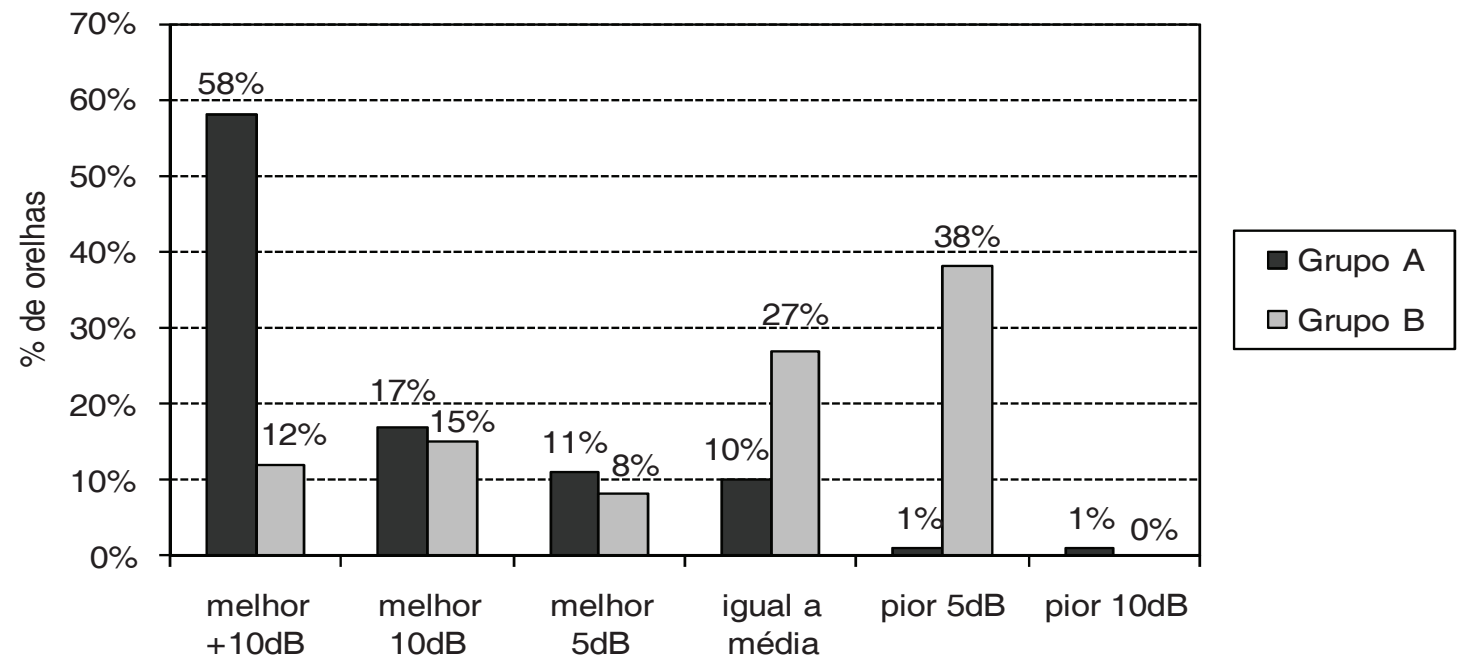

O teste de comparação entre a proporção aplicado nos Grupos A e B para as categorias reunidas (pior $5 \mathrm{~dB}$ e igual a média) resultou nas proporções 0,2333 ou 23,33\% (Grupo A) e 0,6333 ou 63,33\% (Grupo B) sendo $p=0,0000$, ou seja, houve diferença significativa

Figura 2 - Comparação dos resultados do LDV nos grupos A e B 
$73,5 \%$ da amostra (44 orelhas) tiveram resultados dentro destes padrões conforme demonstrado na Figura 2.

Observou-se que $26,5 \%$ da amostra (16 oreIhas) deste mesmo grupo apresentou LDV em intensidade muito melhor do que o esperado. Em sete orelhas $(11,5 \%)$ o resultado ficou categorizado em melhor $+10 \mathrm{~dB}$ e em nove orelhas $(15 \%)$ o resultado ficou melhor $10 \mathrm{~dB}$, mesmo tendo sido eliminado o fator "examinador". Tal fato pode ser explicado em virtude de que, quando se utiliza sinais de fala para avaliar a percepção auditiva, os estímulos têm amplo espectro de freqüência, o que facilita o processo de decodificação dos sons. Decodificar significa identificar um determinado som e reconhecê-lo como estímulo de fala ${ }^{19}$. Tal tarefa ocorre em nível de sistema auditivo central e é dependente de habilidades auditivas como fechamento auditivo, discriminação e memória auditiva. Estas habilidades podem estar interferindo no processo de avaliação quando se trata da logoaudiometria.

No grupo $A$, submetido ao teste à viva voz, observou-se que apenas $23 \%$ da amostra (14 oreIhas) apresentaram um desempenho de LDV compatível com o estimado pela literatura conforme se observa na Figura 1 e $75,5 \%$ (45 orelhas) apresentaram LDV em intensidade muito melhor do que o esperado, ou seja, em 35 orelhas $(58,5 \%)$ o resultado ficou categorizado em melhor $+10 \mathrm{~dB}$ e em 10 orelhas $(17 \%)$ o resultado ficou melhor $10 \mathrm{~dB}$. Neste caso, como o teste foi aplicado à viva voz, o fator "examinador" não foi eliminado, o que permite inferir que aquele que aplica o teste está influenciando o seu resultado.

A prática clínica explica esta constatação. Há momentos em que se torna difícil manter a mesma intensidade de voz, principalmente se a aplicação do teste for longa. As flutuações da intensidade da voz podem ser controladas por intermédio do VU meter do audiômetro, porém, apesar deste recurso, é comum o examinador aumentar ou diminuir a intensidade vocal, sem perceber, "ajudando", desta forma, o indivíduo sob teste a responder em um falso limiar. O profissional que aplica exames logoaudiométricos a viva voz deve ser muito treinado para evitar estas interferências, e por isso estudos sugerem o uso de material gravado ${ }^{18}$.

A diferença significativa observada quando se compara os resultados dos testes aplicados por meio de material gravado e à viva voz, está de acordo com a literatura pesquisada ${ }^{11-13}$. Tal dado permite inferir que a utilização de material gravado na determinação do LDV deve ser priorizada pelos profissionais da área da saúde que se dedicam ao diagnóstico dos distúrbios da audição.

\section{CONCLUSÃO}

Os dados deste estudo permitem determinar que, ao utilizar-se material gravado para o estabelecimento do LDV em curvas de configuração plana, considere-se como padrão de normalidade níveis de intensidade iguais à média tritonal ou pior em $5 \mathrm{~dB}$.

Observou-se que existe diferença estatisticamente significante quando se comparou os resultados do LDV aplicado à viva voz com os resultados alcançados mediante material de fala gravado. Sua utilização, portanto, reduz a possibilidade de interferências externas sobre os resultados do LDV permitindo assim uma maior confiabilidade do teste.

\begin{abstract}
Purpose: to compare the results of the test referred to as voice detection lower thresholds, using a previously-recorded list of trisyllabic words and in live voice in individuals with flat configuration audiogram. Methods: 60 subjects (120 ears) took part in the study, each with an audiometric exam within normal patterns and tonal audiometry at a flat configuration, without auditory incidents, in the age group going from 20 to 30 . The test was given with a live voice and with recorded material, in an acoustically-managed booth, by means of an AC40 audiometer and with the aid of a linked CD player. Results: voice detection lower thresholds were found to be $10 \mathrm{~dB}$ better than the tritonal average in 35 ears $(57 \%$ of the sample) when accomplished by live voice and in 10 ears $(17 \%)$ when accomplished by recorded material; and were found to be $5 \mathrm{~dB}$ worse than the tritonal average in one ear $(3 \%)$ when accomplished by live voice and in 23 ears (38\%) by recorded material. Conclusion: a) when using recorded material for the establishment of LDV in flat configuration curves, one should consider intensity levels equal to the tritonal average or $5 \mathrm{~dB}$ lower as standards of normality and b) a difference was revealed between the results obtained with recorded material and those from live voices, demonstrating the importance of using recorded tests for determining lower auditory thresholds in logoaudiometry.
\end{abstract}

KEYWORDS: Audiometry, Speech; Hearing; Auditory Perception 


\section{REFERÊNCIAS}

1. Wilson $\mathrm{RH}$, Strouse AL. Audiometria com estímulos de fala. In: Musiek FE, Rintelmann WF. Perspectivas atuais em avaliação auditiva. São Paulo: Manole; 2001. p. 21-56.

2. Farfán RC, Solis FF, Palácio J. Evaluación de familiaridade y rendimiento de palabras usadas em logoaudiometría. Rev Chil Tecnol. Med. 2002; 22:984-92.

3. Moreira RR, Ferreira Junior M. Testes de fala: aplicação em portadores de perda auditiva por ruído. Pró-Fono. 2004; 16(3):293-300.

4. Oramas C, Rodriguez W, Acosta M, Arellana AE, Casas AM, Núñez MP, etal. Pruebalogoaudiométrica IU-PLI. Acta Otorrinolaringol Cirurg Cabeza Cuello. 1998; 26(3):191-200.

5. Oliveira MG, Corona AP. Limiar de recepção de fala nas diferentes configurações audiométricas. Rev Fonoaudiol Brasil. 2006; 4:1-2.

6. Russo ICP, Behlau M. Percepção da fala: análise acústica. São Paulo: Lovise; 1993. 57 p.

7. Russo ICP, Santos TMM. Logoaudiometria. In: Russo ICP, Santos TMM. A prática da audiologia clínica. São Paulo: Cortez; 1993. p. 81-98.

8. Redondo MC, Lopes Filho O. Testes básicos da avaliação auditiva. In: Lopes-Filho O. Tratado de fonoaudiologia. São Paulo: Tecmed; 2005. p. 89-110.

9. Penrod JP. Testes de discriminação vocal. In: Katz J. Tratado de audiologia clínica. São Paulo: Manole; 1999. p. 234-55.

10. Harris RW, Goffi-Gomez MVS, Pedalini MEB, Merrill A, Gygi MA. Reconhecimento de palavras dissilábicas psicometricamente equivalentes no Português brasileiro faladas por indivíduos do sexo masculino e do sexo feminino. Pró-Fono. 2001; 13(2):249-62.

11. Carhart R. Monitored live-voice as a test of auditory acuity. J Acoust Soc Am. 1946; 17:338-49. 12. Almeida CIR, Caetano MHU. Logoaudiometria utilizando sentenças sintéticas. Rev Bras Otorrinolaingol. 1988; 54(3):68-72.

13. Kruel EJ, Bell DW, Nixon JC. Factors affecting speech discrimination test difficulty. J Speech Hear Res. 1969; 12(2):281-7.

14. Soncini F, Costa M, Oliveira TMT. Correlação entre limiares de reconhecimento de sentenças no silêncio e limiares tonais. Rev Bras Otorrinolaingol. 2003; 69(5):672-7.

15. Brandy WT. Reliability of voice testes of speech discrimination. J Speech Hear Res. 1966; 9:461-5.

16. Freitas CD, Costa MJ. Variabilidade dos limiares de reconhecimento de fala no teste-reteste de indivíduos normo-ouvintes. Fono Atual. 2006; 8(35):30-40.

17. Harris RW, Goffi MVS, Pedalini MEB, Gygi MA, Merrill A. Palavras trissilábicas psicometricamente equivalentes faladas por indivíduos do sexo feminino e masculino. Pró-Fono. 2001; 13(1):37-53.

18. ISO 8253-3 - NORMA; 1996. Acoustics: audiometric test methods. Part 3: speech audiometry.

19. Ribas A, Lewis DR. O perfil audiológico central de um grupo de crianças portadoras de distúrbio de aprendizagem. Rev Soc Bras Fonoaudiol. 2002; 2:37-41.
RECEBIDO EM: 05/10/2007

ACEITO EM: 21/05/2008

Endereço para correspondência:

Rua Martin Afonso, 2942 ap. 601

Curitiba - PR

CEP: 80730-030

Tel/fax: (41) 3331-7833

E-mail: angela.ribas@utp.br 